\title{
JUCYS-MURPHY ELEMENTS AND CENTRES OF CELLULAR ALGEBRAS
}

\author{
YANBO LI
}

\author{
(Received 30 April 2011)
}

\begin{abstract}
Let $R$ be an integral domain and $A$ a cellular algebra over $R$ with a cellular basis $\left\{C_{S, T}^{\lambda} \mid \lambda \in \Lambda\right.$ and $S, T \in M(\lambda)\}$. Suppose that $A$ is equipped with a family of Jucys-Murphy elements which satisfy the separation condition in the sense of Mathas ['Seminormal forms and Gram determinants for cellular algebras', J. reine angew. Math. 619 (2008), 141-173, with an appendix by M. Soriano]. Let $K$ be the field of fractions of $R$ and $A_{K}=A \bigotimes_{R} K$. We give a necessary and sufficient condition under which the centre of $A_{K}$ consists of the symmetric polynomials in Jucys-Murphy elements. We also give an application of our result to Ariki-Koike algebras.
\end{abstract}

2010 Mathematics subject classification: primary 16U70; secondary 16G30.

Keywords and phrases: Jucys-Murphy elements, cellular algebras, centres.

\section{Introduction}

Jucys-Murphy elements were first constructed for the group algebras of symmetric groups. The combinatorics of these elements allow one to compute simple representations explicitly and often easily in the semisimple case. Then Dipper, James and Murphy [3-7] did a lot of work on representations of Hecke algebras and produced analogues of the Jucys-Murphy elements for Hecke algebras of types A and B. The constructions for other algebras can be found, for example, in [12, 17]. In [4], Dipper and James proved that the centre of a semisimple Hecke algebra of type A consists of symmetric polynomials in Jucys-Murphy elements. A similar result for a Hecke algebra of type A over the ring of Laurent polynomials $\mathbb{Z}\left[q, q^{-1}\right]$ was proved by Francis and Graham [8] in 2006. In [2], Brundan proved that the centre of each degenerate cyclotomic Hecke algebra consists of symmetric polynomials in the Jucys-Murphy elements. An analogous conjecture for Ariki-Koike Hecke algebras is open in the nonsemisimple case.

Cellular algebras were introduced by Graham and Lehrer [11] in 1996, motivated by previous work of Kazhdan and Lusztig [14]. The theory of cellular algebras provides

This work is partially supported by the Research Fund of Doctor Program of Higher Education, Ministry of Education of China.

(C) 2011 Australian Mathematical Publishing Association Inc. 0004-9727/2011 \$16.00 
a systematic framework for studying the representation theory of nonsemisimple algebras which are deformations of semisimple ones. Many classes of algebras from mathematics and physics are found to be cellular, including Hecke algebras of finite type, Ariki-Koike Hecke algebras, $q$-Schur algebras, Brauer algebras, partition algebras, Birman-Wenzl algebras and so on; see [9, 11, 19, 20] for details.

The fact that most of the algebras which have Jucys-Murphy elements are cellular leads one to define Jucys-Murphy elements for general cellular algebras. In [16], Mathas did some work in this direction. Goodman gave another abstraction of JucysMurphy elements in [10]. One of Mathas' results is that if a cellular algebra has a family of Jucys-Murphy elements, then the symmetric polynomials in Jucys-Murphy elements are central under certain conditions. Using the definition of Mathas, in this note we investigate the relations between the centres and the Jucys-Murphy elements of cellular algebras. Note that we also studied centres of symmetric cellular algebras in [15].

Let $R$ be an integral domain and $A$ a cellular $R$-algebra with a cellular basis $\left\{C_{S, T}^{\lambda} \mid S, T \in M(\lambda), \lambda \in \Lambda\right\}$. Let $K$ be the field of fractions of $R$ and $A_{K}=A \bigotimes_{R} K$. Suppose that $A$ is equipped with a family of Jucys-Murphy elements $L_{1}, \ldots, L_{m}$ which satisfy the separation condition [16]. We will give a necessary and sufficient condition under which the centre of $A_{K}$ consists of the symmetric polynomials in Jucys-Murphy elements. This condition is also a necessary condition for the centre of $A$ consisting of the symmetric polynomials in Jucys-Murphy elements. Moreover, by this result, we prove that the centres of Ariki-Koike Hecke algebras consist of the symmetric polynomials in Jucys-Murphy elements in the semisimple case. The proof is different from Ariki's in [1] and Ram's in [18].

We start with a quick review of the results under the separation condition in [16]. Then we prove the main result in Section 3. Finally, we give an application of our result by Ariki-Koike algebras in Section 4.

\section{Cellular algebras and Jucys-Murphy elements}

In this section, we first recall the definition of cellular algebras and then give a quick review of the results under the so-called separation condition in Mathas' paper [16].

Definition 2.1 [11, Definition 1.1]. Let $R$ be a commutative ring with identity. An associative unital $R$-algebra is called a cellular algebra with cell datum $(\Lambda, M, C, i)$ if the following conditions are satisfied.

(C1) The finite set $\Lambda$ is a poset. Associated with each $\lambda \in \Lambda$, there is a finite set $M(\lambda)$. The algebra $A$ has an $R$-basis $\left\{C_{S, T}^{\lambda} \mid S, T \in M(\lambda), \lambda \in \Lambda\right\}$.

(C2) The map $i$ is an $R$-linear anti-automorphism of $A$ with $i^{2}=i d$ which sends $C_{S, T}^{\lambda}$ to $C_{T, S}^{\lambda}$.

(C3) If $\lambda \in \Lambda$ and $S, T \in M(\lambda)$, then for any element $a \in A$, we have

$$
a C_{S, T}^{\lambda} \equiv \sum_{S^{\prime} \in M(\lambda)} r_{a}\left(S^{\prime}, S\right) C_{S^{\prime}, T}^{\lambda}(\bmod A(<\lambda)),
$$


where $r_{a}\left(S^{\prime}, S\right) \in R$ is independent of $T$ and where $A(<\lambda)$ is the $R$-submodule of $A$ generated by $\left\{C_{S^{\prime \prime}, T^{\prime \prime}}^{\mu} \mid S^{\prime \prime}, T^{\prime \prime} \in M(\mu), \mu<\lambda\right\}$. Applying $i$ to the equation in (C3),

$$
C_{T, S}^{\lambda} i(a) \equiv \sum_{S^{\prime} \in M(\lambda)} r_{a}\left(S^{\prime}, S\right) C_{T, S^{\prime}}^{\lambda}(\bmod A(<\lambda)) .
$$

Let $R$ be an integral domain. Given a cellular algebra $A$, we will also assume that $M(\lambda)$ is a poset with an order $\leq_{\lambda}$. Let $M(\Lambda)=\bigsqcup_{\lambda \in \Lambda} M(\lambda)$. We consider $M(\Lambda)$ as a poset with an order $\leq$ as follows.

$$
S \leq T \Leftrightarrow \begin{cases}S \leq_{\lambda} T, & \text { if } S, T \in M(\lambda) \\ \lambda \leq \mu, & \text { if } S \in M(\lambda), T \in M(\mu) .\end{cases}
$$

Let $K$ be the field of fractions of $R$ and $A_{K}=A \bigotimes_{R} K$. We will consider $A$ as a subalgebra of $A_{K}$.

Definition 2.2 [16, Definition 2.4]. Let $R$ be an integral domain and $A$ a cellular algebra. A family $L_{1}, \ldots, L_{m}$ of elements of $A$ is called a family of Jucys-Murphy elements if:

(1) $L_{i} L_{j}=L_{j} L_{i}$, for $1 \leq i, j \leq m$;

(2) $i\left(L_{j}\right)=L_{j}$, for $j=1, \ldots, m$;

(3) For all $\lambda \in \Lambda, S, T \in M(\lambda)$ and $L_{i}, i=1, \ldots, m$,

$$
C_{S, T}^{\lambda} L_{i} \equiv c_{T}(i) C_{S, T}^{\lambda}+\sum_{V<T} r_{L_{i}}(V, T) C_{S, V}^{\lambda}(\bmod A(<\lambda)),
$$

where $c_{T}(i) \in R, r_{L_{i}}(T, V) \in R$. We call $c_{T}(i)$ the content of $T$ at $i$. Denote $\left\{c_{T}(i) \mid T \in M(\Lambda)\right\}$ by $\mathscr{C}(i)$ for $i=1,2, \ldots, m$.

ExAmple 2.3. Let $K$ be a field. Let $A$ be the group algebra of the symmetric group $S_{n}$. Set $L_{i}=\sum_{j=1}^{i-1}(i, j)$ for $i=2, \ldots, n$. Then $L_{i}, i=2, \ldots, n$, is a family of Jucys-Murphy elements of $A$.

Definition 2.4 [16, Definition 2.8]. Let $A$ be a cellular algebra with a family of JucysMurphy elements $\left\{L_{1}, \ldots, L_{m}\right\}$. We say that the Jucys-Murphy elements satisfy the separation condition if for any $S, T \in M(\Lambda), S \leq T, S \neq T$, there exists some $i$ with $1 \leq i \leq m$ such that $c_{S}(i) \neq c_{T}(i)$.

Remark 2.5. The separation condition forces $A_{K}$ to be semisimple (see [16]).

From now on, we always assume that $A$ is a cellular algebra equipped with a family of Jucys-Murphy elements which satisfy the separation condition. We now recall some results of [16].

Definition 2.6 [16, Definition 3.1]. Let $A$ be a cellular algebra with a family of JucysMurphy elements $\left\{L_{1}, \ldots, L_{m}\right\}$. For $\lambda \in \Lambda, S, T \in M(\lambda)$, define

$$
F_{T}=\prod_{i} \prod_{c \in \mathscr{C}(i), c \neq c_{T}(i)}\left(L_{i}-c\right) /\left(c_{T}(i)-c\right)
$$

and $f_{S, T}^{\lambda}=F_{S} C_{S, T}^{\lambda} F_{T}$. 
Note that the coefficient of $C_{S, T}^{\lambda}$ in the expansion of $f_{S, T}^{\lambda}$ is 1 for any $\lambda \in \Lambda$ and $S, T \in M(\lambda)$, see [16, 3.3(a)]. Then Mathas proved the following theorems.

Theorem 2.7 [16, Theorem 3.7]. Let $A$ be a cellular algebra with a family of JucysMurphy elements $\left\{L_{1}, \ldots, L_{m}\right\}$. Let $\lambda, \mu \in \Lambda, S, T \in M(\lambda)$ and $U, V \in M(\mu)$. Then:

$$
f_{S, T}^{\lambda} f_{U, V}^{\mu}= \begin{cases}\gamma_{T} f_{S, V}^{\lambda}, & \lambda=\mu, T=U, \\ 0, & \text { otherwise, }\end{cases}
$$

where $\gamma_{T} \in K$ and $\gamma_{T} \neq 0$ for all $T \in M(\Lambda)$;

(2) $\left\{f_{S, T}^{\lambda} \mid S, T \in M(\lambda), \lambda \in \Lambda\right\}$ is a cellular basis of $A_{K}$.

Theorem 2.8 [16, Theorem 3.16]. Let A be a cellular algebra with a family of JucysMurphy elements $\left\{L_{1}, \ldots, L_{m}\right\}$.

(1) Let $\lambda \in \Lambda$ and $T \in M(\lambda)$. Then $F_{T}$ is a primitive idempotent in $A_{K}$. Moreover, $\left\{F_{T} \mid T \in M(\lambda)\right\}$ is a complete set of pairwise orthogonal primitive idempotents in $A_{K}$.

(2) $F_{\lambda}=\sum_{T \in M(\lambda)} F_{T}$ is a central idempotent in $A_{K}$ for any $\lambda \in \Lambda$. Moreover, $\left\{F_{\lambda} \mid \lambda \in \Lambda\right\}$ is a complete set of central idempotents which are primitive in $Z\left(A_{K}\right)$.

(3) In particular, $1=\sum_{\lambda \in \Lambda} F_{\lambda}=\sum_{T \in M(\Lambda)} F_{T}$ and $L_{i}=\sum_{T \in M(\Lambda)} c_{T}(i) F_{T}$.

\section{Jucys-Murphy elements and centres of cellular algebras}

In [16], Mathas gave a relation between the centre and Jucys-Murphy elements of a cellular algebra.

Proposition 3.1 [16, Proposition 4.13]. Let A be a cellular algebra with JucysMurphy elements $\left\{L_{1}, \ldots, L_{m}\right\}$. Suppose that for $\lambda \in \Lambda$, there exist scalars $c_{\lambda}(i)$, for $1 \leq i \leq m$, such that

$$
\left\{c_{\lambda}(i) \mid 1 \leq i \leq m\right\}=\left\{c_{T}(i) \mid 1 \leq i \leq m\right\}
$$

for any $T \in M(\lambda)$. Then every symmetric polynomial in $L_{1}, \ldots, L_{m}$ belongs to the centre of $A_{K}$.

In fact, the converse proposition also holds.

Proposition 3.2. Let A be a cellular algebra with a family of Jucys-Murphy elements $\left\{L_{1}, \ldots, L_{m}\right\}$. Suppose that every symmetric polynomial in $L_{1}, \ldots, L_{m}$ belongs to the centre of $A_{K}$. Then for any $\lambda \in \Lambda$, there exist scalars $c_{\lambda}(i), 1 \leq i \leq m$, such that

$$
\left\{c_{\lambda}(i) \mid 1 \leq i \leq m\right\}=\left\{c_{T}(i) \mid 1 \leq i \leq m\right\}
$$

for any $T \in M(\lambda)$.

Proof. Suppose that there exist some $\lambda \in \Lambda$ and $S, T \in M(\lambda)$ such that

$$
\left\{c_{S}(i) \mid 1 \leq i \leq m\right\} \neq\left\{c_{T}(i) \mid 1 \leq i \leq m\right\}
$$


Then there exists a symmetric polynomial $p$ such that

$$
p\left(c_{S}(1), \ldots, c_{S}(m)\right) \neq p\left(c_{T}(1), \ldots, c_{T}(m)\right) .
$$

Note that $L_{i}=\sum_{X \in M(\Lambda)} c_{X}(i) F_{X}$. Then

$$
p\left(L_{1}, \ldots, L_{m}\right)=\sum_{U \in M(\Lambda)} p\left(c_{U}(1), \ldots, c_{U}(m)\right) F_{U} .
$$

By Theorem 2.8(1), multiplying by $F_{T}$ on both sides, we get

$$
p\left(L_{1}, \ldots, L_{m}\right) F_{T}=p\left(c_{T}(1), \ldots, c_{T}(m)\right) F_{T} .
$$

The equality $p\left(L_{1}, \ldots, L_{m}\right) F_{S}=p\left(c_{S}(1), \ldots, c_{S}(m)\right) F_{S}$ is obtained similarly.

On the other hand, since $p\left(L_{1}, \ldots, L_{m}\right) \in Z\left(A_{K}\right)$, then by Theorem 2.8(3)

$$
p\left(L_{1}, \ldots, L_{m}\right)=\sum_{\lambda \in \Lambda} r_{\lambda} F_{\lambda}
$$

where $r_{\lambda} \in K$. Multiplying by $F_{T}$ on both sides of this equation

$$
p\left(L_{1}, \ldots, L_{m}\right) F_{T}=r_{\lambda} F_{T} .
$$

The equation $p\left(L_{1}, \ldots, L_{m}\right) F_{S}=r_{\lambda} F_{S}$ can be obtained similarly. Then

$$
p\left(c_{T}(1), \ldots, c_{T}(m)\right)=r_{\lambda}=p\left(c_{S}(1), \ldots, c_{S}(m)\right),
$$

which is a contradiction.

Now we are in a position to give the main result of this paper.

THeOREM 3.3. Let $R$ be an integral domain and A a cellular $R$-algebra with a cellular basis $\left\{C_{S, T}^{\lambda} \mid S, T \in M(\lambda), \lambda \in \Lambda\right\}$. Let $K$ be the field of fractions of $R$ and $A_{K}=$ $A \bigotimes_{R} K$. Suppose that $A$ is equipped with a family of Jucys-Murphy elements $L_{1}, \ldots, L_{m}$ which satisfy the separation condition and all symmetric polynomials in $L_{1}, \ldots, L_{m}$ belong to the centre of $A_{K}$. Then the following are equivalent.

(1) The centre of $A_{K}$ consists of all symmetric polynomials in the Jucys-Murphy elements.

(2) For any $\lambda, \mu \in \Lambda$ with $\lambda \neq \mu$,

$$
\left\{c_{\lambda}(i) \mid 1 \leq i \leq m\right\} \neq\left\{c_{\mu}(i) \mid 1 \leq i \leq m\right\} .
$$

To prove this theorem, we need the following two lemmas.

Lemma 3.4. Let $X_{1}, X_{2}, \ldots, X_{m}$ be indeterminates over a field $K$ and let $\left\{x_{1}, \ldots, x_{m}\right\}$ and $\left\{y_{1}, \ldots, y_{m}\right\}$ be two families of elements in $K$. Suppose that there exists some $k \in K$, such that $p\left(x_{1}, \ldots, x_{m}\right)=k p\left(y_{1}, \ldots, y_{m}\right)$ for any symmetric polynomial $p\left(X_{1}, X_{2}, \ldots, X_{m}\right) \in K\left[X_{1}, X_{2}, \ldots, X_{m}\right]$. Then $\left\{x_{1}, \ldots, x_{m}\right\}=\left\{y_{1}, \ldots, y_{m}\right\}$. 
Proof. Clearly, if $p$ is a symmetric polynomial, then $p^{2}$ is also a symmetric polynomial. Then

$$
\left(p\left(x_{1}, \ldots, x_{m}\right)\right)^{2}=\left(k p\left(y_{1}, \ldots, y_{m}\right)\right)^{2}=k\left(p\left(y_{1}, \ldots, y_{m}\right)\right)^{2} .
$$

Hence $\left(k^{2}-k\right)\left(p\left(y_{1}, \ldots, y_{m}\right)\right)^{2}=0$. Then $k^{2}-k=0$ since $p$ is arbitrary. So we have $k=0$ or $k=1$. If $k=0$, then $p\left(x_{1}, \ldots, x_{m}\right)=0$ for any $p$. This is impossible. Then $k=1$. That is, $p\left(x_{1}, \ldots, x_{m}\right)=p\left(y_{1}, \ldots, y_{m}\right)$ for arbitrary $p$.

Let $\left\{k_{11}, \ldots, k_{1 m}\right\}, \ldots,\left\{k_{n 1}, \ldots, k_{n m}\right\}$ be $n$ families of elements in $K$ and $p$ a symmetric polynomial. We will denote $p\left(k_{i 1}, \ldots, k_{i m}\right)$ by $p(i)$.

Lemma 3.5. Suppose that $\left\{k_{11}, \ldots, k_{1 m}\right\}, \ldots,\left\{k_{n 1}, \ldots, k_{n m}\right\}$ are $n$ families of elements in a field $K$ and $X_{1}, X_{2}, \ldots, X_{m}$ indeterminates. Let $p_{1}^{\prime}\left(X_{1}, X_{2}, \ldots, X_{m}\right), \ldots, p_{n}^{\prime}$ $\left(X_{1}, X_{2}, \ldots, X_{m}\right) \in K\left[X_{1}, X_{2}, \ldots, X_{m}\right]$ be $n$ symmetric polynomials such that

$$
\left|\begin{array}{ccc}
p_{1}^{\prime}(1) & \ldots & p_{1}^{\prime}(n) \\
\ldots & \ldots & \ldots \\
p_{n}^{\prime}(1) & \ldots & p_{n}^{\prime}(n)
\end{array}\right| \neq 0
$$

Then there exist $n$ symmetric polynomials $p_{1}, \ldots, p_{n}$ such that

$$
\left|\begin{array}{cccc}
p_{1}(1) & p_{1}(2) & \ldots & p_{1}(n) \\
0 & p_{2}(2) & \ldots & p_{2}(n) \\
\ldots & \ldots & \ldots & \ldots \\
0 & 0 & \ldots & p_{n}(n)
\end{array}\right| \neq 0
$$

PRoof. The proof of the lemma follows from the facts that elementary row operations do not change the determinant up to multiplication by \pm 1 and linear combinations of symmetric polynomials are still symmetric polynomials.

Proof of Theorem. Since $p\left(L_{1}, L_{2}, \ldots, L_{m}\right)=\sum_{\lambda \in \Lambda} p\left(c_{\lambda}(1), \ldots, c_{\lambda}(m)\right) F_{\lambda}$ (see the proof of [16, Proposition 4.13]), then $(1) \Rightarrow(2)$ is obvious.

We now prove $(2) \Rightarrow(1)$. Suppose that there are $n$ elements in $\Lambda$. Without loss of generality, extend the partial order on $\Lambda$ to a total one and denote the elements in $\Lambda$ by $1,2, \ldots, n$.

If there exist symmetric polynomials $p_{1}, p_{2}, \ldots, p_{n}$ such that

$$
\left|\begin{array}{cccc}
p_{1}(1) & p_{1}(2) & \ldots & p_{1}(n) \\
p_{2}(1) & p_{2}(2) & \ldots & p_{2}(n) \\
\ldots & \ldots & \ldots & \ldots \\
p_{n}(1) & p_{n}(2) & \ldots & p_{n}(n)
\end{array}\right| \neq 0
$$

where $p_{i}(j)=p_{i}\left(c_{j}(1), c_{j}(2), \ldots, c_{j}(m)\right)$, then by

$$
p\left(L_{1}, L_{2}, \ldots, L_{m}\right)=\sum_{\lambda \in \Lambda} p\left(c_{\lambda}(1), \ldots, c_{\lambda}(m)\right) F_{\lambda},
$$


any $F_{\lambda}$ is a linear combination of symmetric polynomials in Jucys-Murphy elements. Note that the algebra $A_{K}$ is semisimple and then $\left\{F_{\lambda} \mid \lambda \in \Lambda\right\}$ is a basis of the centre of $A_{K}$; thus the set of symmetric polynomials in Jucys-Murphy elements coincides with $Z\left(A_{K}\right)$.

Hence the key is to prove the existence of those $n$ eligible polynomials. Clearly, there exists a symmetric polynomial $p_{1}$ such that $p_{1}(1) \neq 0$,

For any $1 \leq i<n$, assume that there exist symmetric polynomials $p_{1}^{\prime}, p_{2}^{\prime}, \ldots, p_{i}^{\prime}$ such that

$$
\left|\begin{array}{ccc}
p_{1}^{\prime}(1) & \ldots & p_{1}^{\prime}(i) \\
\ldots & \ldots & \ldots \\
p_{i}^{\prime}(1) & \ldots & p_{i}^{\prime}(i)
\end{array}\right| \neq 0 .
$$

Then by Lemma 3.5, there exist symmetric polynomials $p_{1}, \ldots, p_{i}$ such that

$$
\left|\begin{array}{cccc}
p_{1}(1) & p_{1}(2) & \ldots & p_{1}(i) \\
0 & p_{2}(2) & \ldots & p_{2}(i) \\
\ldots & \ldots & \ldots & \ldots \\
0 & 0 & \ldots & p_{i}(i)
\end{array}\right| \neq 0
$$

Suppose that for any symmetric polynomial $p$,

$$
d:=\left|\begin{array}{ccccc}
p_{1}(1) & p_{1}(2) & \ldots & p_{1}(i) & p_{1}(i+1) \\
0 & p_{2}(2) & \ldots & p_{2}(i) & p_{2}(i+1) \\
\ldots & \ldots & \ldots & \ldots & \ldots \\
0 & 0 & \ldots & p_{i}(i) & p_{i}(i+1) \\
p(1) & p(2) & \ldots & p(i) & p(i+1)
\end{array}\right|=0 .
$$

Then $p(i+1)=k_{1} p(1)+k_{2} p(2)+\cdots+k_{i} p(i)$, where $k_{j} \in K$ is independent of $p$ for $j=1, \ldots, i$. Note that $p_{i} p$ is also a symmetric polynomial, and we have

$$
p_{i} p(i+1)=k_{1} p_{i} p(1)+k_{2} p_{i} p(2)+\cdots+k_{i} p_{i} p(i) .
$$

Assume that $p_{i}(i+1) \neq 0$. Then $p_{i}(i+1) p(i+1)=k_{i} p_{i}(i) p(i)$, or $p(i+1)=k p(i)$, where $k \in K$ is independent of the choice of $p$. This implies that $p(i+1)=p(i)$ by Lemma 3.4, which is a contradiction. Then $p_{i}(i+1)=0$. That is, $k_{i} p_{i}(i) p(i)=0$. Since $p_{i}(i) \neq 0$ and $p$ is arbitrary, then $k_{i}=0$. Repeating this process similarly, we have $k_{j}=0$ for $j=1, \ldots, i$ and then $p(i+1)=0$. But this is impossible for $p$ is arbitrary. Then there exists a symmetric polynomial $p$ such that $d \neq 0$. This implies that the eligible $n$ polynomials exist and the proof is complete.

Corollary 3.6. Let $R$ be an integral domain and A a cellular algebra. Suppose that A is equipped with a family of Jucys-Murphy elements which separate A. If the centre of A consists of symmetric polynomials in Jucys-Murphy elements, then

$$
\left\{c_{\lambda}(i) \mid 1 \leq i \leq m\right\} \neq\left\{c_{\mu}(i) \mid 1 \leq i \leq m\right\},
$$

for arbitrary $\lambda, \mu \in \Lambda$ with $\lambda \neq \mu$. 


\section{An application to Ariki-Koike Hecke algebras}

In this section, we prove that the centre of a semisimple Ariki-Koike Hecke algebra $(q \neq 1)$ consists of the symmetric polynomials in Jucys-Murphy elements. This is a new proof, different from Ariki's in [1] and Ram's [18].

Firstly, we recall some notions of combinatorics. Recall that a partition of $n$ is a nonincreasing sequence of nonnegative integers $\lambda=\left(\lambda_{1}, \ldots, \lambda_{r}\right)$ such that $\sum_{i=1}^{r} \lambda_{i}=n$. The diagram of a partition $\lambda$ is the subset $[\lambda]=\left\{(i, j) \mid 1 \leq j \leq \lambda_{i}, i \geq 1\right\}$. The elements of $\lambda$ are called nodes. Define the residue of the node $(i, j) \in[\lambda]$ to be $j-i$. For any partition $\lambda=\left(\lambda_{1}, \lambda_{2}, \ldots\right)$, the conjugate of $\lambda$ is defined to be a partition $\lambda^{\prime}=$ $\left(\lambda_{1}^{\prime}, \lambda_{2}^{\prime}, \ldots\right)$, where $\lambda_{j}^{\prime}$ is equal to the number of nodes in column $j$ of $[\lambda]$ for $j=1,2, \ldots$ For partitions, the following lemma is clear.

Lemma 4.1. Let $\lambda$ and $\mu$ be two partitions of $n$. Then $\lambda=\mu$ if and only if all residues of nodes in $[\lambda]$ and $[\mu]$ are the same.

Given two partitions $\lambda$ and $\mu$ of $n$, write $\lambda \unrhd \mu$ if

$$
\sum_{i=1}^{j} \lambda_{i} \geq \sum_{i=1}^{j} \mu_{i}, \quad \text { for all } i \geq 1 .
$$

This is the so-called dominance order.

A $\lambda$-tableau is a bijection $\mathrm{t}:[\lambda] \rightarrow\{1,2, \ldots, n\}$. We say that $\mathrm{t}$ is a standard $\lambda$ tableau if the entries in $t$ increase from left to right in each row and from top to bottom in each column. Denote by $\mathrm{t}^{\lambda}$ (respectively, $\mathrm{t}_{\lambda}$ ) the standard $\lambda$-tableau, in which the numbers $1,2, \ldots, n$ appear in order along successive rows (respectively, columns). The row stabilizer of $\mathrm{t}^{\lambda}$, denoted by $S_{\lambda}$, is the standard Young subgroup of $S_{n}$ corresponding to $\lambda$. Let $\operatorname{Std}(\lambda)$ be the set of all standard $\lambda$-tableaux.

For a fixed positive integer $m$, an $m$-multipartition of $n$ is an $m$-tuple of partitions which sum to $n$. Let

$$
\lambda=\left(\left(\lambda_{11}, \lambda_{12}, \ldots, \lambda_{1 i_{1}}\right),\left(\lambda_{21}, \lambda_{22}, \ldots, \lambda_{2 i_{2}}\right), \ldots,\left(\lambda_{m 1}, \lambda_{m 2}, \ldots, \lambda_{m i_{m}}\right)\right)
$$

be an $m$-multipartition of $n$; we denote $\lambda_{j 1}+\lambda_{j 2}+\cdots+\lambda_{j i}$ by $n_{j \lambda}$ for $1 \leq j \leq m$. A standard $\lambda$-tableau is an $m$-tuple of standard tableaux. We can define $\mathrm{t}^{\lambda}$ similarly.

Let $R$ be an integral domain, $q, u_{1}, u_{2}, \ldots, u_{m} \in R$ and $q$ invertible. Fix two positive integers $n$ and $m$. Then an Ariki-Koike algebra $\mathscr{H}_{n, m}$ is the associative $R$-algebra with generators $T_{0}, T_{1}, \ldots, T_{n-1}$ and relations

$$
\begin{gathered}
\left(T_{0}-u_{1}\right)\left(T_{0}-u_{2}\right) \cdots\left(T_{0}-u_{m}\right)=0, \\
T_{0} T_{1} T_{0} T_{1}=T_{1} T_{0} T_{1} T_{0}, \\
\left(T_{i}-q\right)\left(T_{i}+1\right)=0, \quad \text { for } 1 \leq i \leq n-1, \\
T_{i} T_{i+1} T_{i}=T_{i+1} T_{i} T_{i+1}, \quad \text { for } 1 \leq i \leq n-2, \\
T_{i} T_{j}=T_{j} T_{i}, \quad \text { for } 0 \leq i<j-1 \leq n-2 .
\end{gathered}
$$


Denote by $\Lambda$ the set of $m$-multipartitions of $n$. For $\lambda \in \Lambda$, let $M(\lambda)$ be the set of standard $\lambda$-tableau. Then $\mathscr{H}_{n, m}$ has a cellular basis of the form $\left\{m_{\mathfrak{s t}}^{\lambda} \mid \lambda \in \Lambda, \mathfrak{s}, t \in M(\lambda)\right\}$. See [6] for details.

Let $L_{i}=q^{1-i} T_{i-1} \cdots T_{1} T_{0} T_{1} \cdots T_{i-1}$. Then $L_{1}, L_{2}, \ldots, L_{n}$ is a family of JucysMurphy elements of $\mathscr{H}_{n, m}$. If $i$ is in row $r$ and column $c$ of the $j$ th tableau of t, then $m_{\mathrm{st}}^{\lambda} L_{i} \equiv u_{j} q^{c-r} m_{\mathrm{st}}^{\lambda}$. If $[1]_{q} \cdots[n]_{q} \prod_{1 \leq i<j \leq m} \prod_{|d|<n}\left(q^{d} u_{i}-u_{j}\right) \neq 0$ and $q \neq 1$, then the Jucys-Murphy elements separate $M(\Lambda)$. These statements were proved in [13].

Denote $\mathscr{H}_{n, m} \otimes_{R} K$ by $\mathscr{H}_{n, m, K}$. The following result was proved in $[1,18]$. We give a new proof here.

THeOREM $4.2[1,18]$. The centre of $\mathscr{H}_{n, m, K}$ is equal to the set of symmetric polynomials in the Jucys-Murphy elements if $[1]_{q} \cdots[n]_{q} \prod_{1 \leq i<j \leq m} \prod_{|d|<n}\left(q^{d} u_{i}-u_{j}\right) \neq 0$ and $q \neq 1$.

Proof. That the algebra $\mathscr{H}_{n, m, K}$ satisfies the conditions of the Proposition 3.1 was pointed out in [16]. By Theorem 3.3, we only need to show that for any $\lambda, \mu \in \Lambda$ with $\lambda \neq \mu,\left\{c_{\lambda}(i) \mid 1 \leq i \leq n\right\} \neq\left\{c_{\mu}(i) \mid 1 \leq i \leq n\right\}$. Note that we can obtain these two sets using $\mathrm{t}^{\lambda}$ and $\mathrm{t}^{\mu}$, respectively.

Case 1. There exists $1 \leq j \leq m$ such that $n_{j \lambda} \neq n_{j \mu}$. Then by the separation condition, the number of elements of the form $u_{j} q^{x}$ is $n_{j \lambda}$ in $\left\{c_{\lambda}(i) \mid 1 \leq i \leq M\right\}$ and is $n_{j \mu}$ in $\left\{c_{\mu}(i) \mid\right.$ $1 \leq i \leq M\}$, where $x \in \mathbb{Z}$. This implies that $\left\{c_{\lambda}(i) \mid 1 \leq i \leq M\right\} \neq\left\{c_{\mu}(i) \mid 1 \leq i \leq n\right\}$.

Case 2. $n_{j \lambda}=n_{j \mu}$ for all $1 \leq j \leq m$. Then there must exist $1 \leq s \leq m$, such that the partition of $n_{s}$ in $\lambda$ is not equal to that in $\mu$ since $\lambda \neq \mu$. Denote the partitions by $\lambda_{s}$ and $\mu_{s}$. Then the residues of $\lambda_{s}$ and $\mu_{s}$ are not the same. Now by Lemma 4.1 and the separation condition, the set of all the elements of the form $u_{s} q^{x}$ in $\left\{c_{\lambda}(i) \mid 1 \leq i \leq M\right\}$ is different from that in $\left\{c_{\mu}(i) \mid 1 \leq i \leq n\right\}$.

REMARK 4.3. If $q \neq 1$, then $[1]_{q} \cdots[n]_{q} \prod_{1 \leq i<j \leq m} \prod_{|d|<n}\left(q^{d} u_{i}-u_{j}\right) \neq 0$ if and only if $\mathscr{H}_{n, m, K}$ is semisimple. See [1] for details.

\section{Acknowledgements}

The author acknowledges his supervisor Professor C. C. Xi. He acknowledges Dr W. Hu and Z. Xiao for many helpful conversations.

\section{References}

[1] S. Ariki, 'On the semi-simplicity of the Hecke algebra of $(\mathbb{Z} / r \mathbb{Z}) \imath S_{n}$ ', J. Algebra 169 (1994), 216-225.

[2] J. Brundan, 'Centers of degenerate cyclotomic Hecke algebras and parabolic category', Represent. Theory 12 (2008), 236-259.

[3] R. Dipper and G. James, 'Representations of Hecke algebras of general linear groups', Proc. London Math. Soc. 52(3) (1986), 20-52.

[4] R. Dipper and G. James, 'Blocks and idempotents of Hecke algebras of general linear groups', Proc. London Math. Soc. 54(3) (1987), 57-82.

[5] R. Dipper and G. James, 'Representations of Hecke algebras of type $B_{n}$ ', J. Algebra 146 (1992), $454-481$. 
[6] R. Dipper, G. James and A. Mathas, 'Cyclotomic q-Schur algebras', Math. Z. 229 (1998), 385-416.

[7] R. Dipper, G. James and G. Murphy, 'Hecke algebras of type $B_{n}$ at roots of unity', Proc. London Math. Soc. 70(3) (1995), 505-528.

[8] A. Francis and J. J. Graham, 'Centers of Hecke algebras: the Dipper-James conjecture', J. Algebra 306 (2006), 244-267.

[9] M. Geck, 'Hecke algebras of finite type are cellular', Invent. Math. 169 (2007), 501-517.

[10] F. Goodman and J. Graber, 'On cellular algebras with Jucys-Murphy elements', J. Algebra 330 (2011), 147-176.

[11] J. J. Graham and G. I. Lehrer, 'Cellular algebras', Invent. Math. 123 (1996), 1-34.

[12] T. Halverson and A. Ram, 'Partition algebras', European J. Combin. 26(6) (2005), 869-921.

[13] G. James and A. Mathas, 'The Jantzen sum formula for cyclotomic $q$-Schur algebras', Trans. Amer. Math. Soc. 352 (2000), 5381-5404.

[14] D. Kazhdan and G. Lusztig, 'Representations of Coxeter groups and Hecke algebras', Invent. Math. 53 (1979), 165-184.

[15] Y. Li, 'Centers of symmetric cellular algebras', Bull. Aust. Math. Soc. 82 (2010), 511-522.

[16] A. Mathas, 'Seminormal forms and Gram determinants for cellular algebras', J. reine angew. Math. 619 (2008), 141-173 With an appendix by M. Soriano.

[17] A. Ram, 'Seminormal representations of Weyl groups and Iwahori-Hecke algebras', Proc. London Math. Soc. 75(3) (1997), 99-133.

[18] A. Ram and J. Ramagge, 'Affine Hecke algebras, cyclotomic Hecke algebras and Clifford theorem', in: A Tribute to C.S. Seshadri: Perspectives in Geometry and Representation Theory, (eds. V. Lakshimibai et al.) (Hindustan Book Agency, New Delhi, 2003), pp. 428-466.

[19] C. C. Xi, 'Partition algebras are cellular', Compositio Math. 119 (1999), 99-109.

[20] C. C. Xi, 'On the quasiheredity of Birman-Wenzl algebras', Adv. Math. 154 (2000), 280-298.

\author{
YANBO LI, Department of Information and Computing Sciences, \\ Northeastern University at Qinhuangdao, Qinhuangdao 066004, PR China \\ and \\ School of Mathematics Sciences, Beijing Normal University, \\ Beijing 100875, PR China \\ e-mail: liyanbo707@163.com
}

